\title{
An Empirical Study of Global Malware Encounters
}

\author{
Ghita Mezzour \\ Electrical and Computer \\ Engineering Department \\ Carnegie Mellon University \\ 5000 Forbes avenue \\ Pittsburgh, PA 15213 \\ mezzour@cmu.edu
}

\author{
Kathleen M. Carley \\ Institute for Software \\ Research \\ Carnegie Mellon University \\ 5000 Forbes avenue \\ Pittsburgh, PA 15213 \\ kathleen.carley@cs.cmu.edu
}

\author{
L. Richard Carley \\ Electrical and Computer \\ Engineering Department \\ Carnegie Mellon University \\ 5000 Forbes avenue \\ Pittsburgh, PA 15213 \\ carley@ece.cmu.edu
}

\begin{abstract}
The number of trojans, worms, and viruses that computers encounter varies greatly across countries. Empirically identifying factors behind such variation can provide a scientific empirical basis to policy actions to reduce malware encounters in the most affected countries. However, our understanding of these factors is currently mainly based on expert opinions, not empirical evidence.

In this paper, we empirically test alternative hypotheses about factors behind international variation in the number of trojan, worm, and virus encounters. We use the Symantec Anti-Virus (AV) telemetry data collected from more than 10 million Symantec customer computers worldwide that we accessed through the Symantec Worldwide Intelligence Environment (WINE) platform. We use regression analysis to test for the effect of computing and monetary resources, web browsing behavior, computer piracy, cyber security expertise, and international relations on international variation in malware encounters.

We find that trojans, worms, and viruses are most prevalent in Sub-Saharan African countries. Many Asian countries also encounter substantial quantities of malware. Our regression analysis reveals that the main factor that explains high malware exposure of these countries is a widespread computer piracy especially when combined with poverty. Our regression analysis also reveals that, surprisingly, web browsing behavior, cyber security expertise, and international relations have no significant effect.
\end{abstract}

\section{Categories and Subject Descriptors}

K.4 [Computers and Society]: Public Policy IssuesAbuse and crime involving computers

\section{General Terms}

Security

Permission to make digital or hard copies of part or all of this work for personal or classroom use is granted without fee provided that copies are not made or distributed for profit or commercial advantage and that copies bear this notice and the full citation on the first page. Copyrights for third-party components of this work must be honored. For all other uses, contact the Owner/Author.

HotSoS '15 April 21 - 22 2015, Urbana, IL, USA

Copyright is held by the owner/author(s).

ACM 978-1-4503-3376-4/15/04.

http://dx.doi.org/10.1145/2746194.2746202

\section{Keywords}

Science of security, social factors, international factors

\section{INTRODUCTION}

Computers in different countries encounter very different quantities of malware. Empirically identifying factors behind such phenomenon can provide a sound basis to policies to reduce malware encounters in countries that encounter disproportionate quantities of malware. Reducing malware encounters in these countries is likely to benefit end-users worldwide given that we live in an inter-connected world.

Many studies notice international differences in malware encounters $[44,26,27,8,9]$, but mostly hypothesize about reasons behind such differences without empirically testing the validity of such hypotheses. For example, Caballero et al. [8] hypothesize that fake anti-viruses are most prevalent in Western European and North American countries because attackers are interested in taking advantage of the large computing and monetary resources in these countries. The main exception is the work by Mezzour et al. [27] that statistically tests hypotheses behind international variation in the number of exploits, web attacks, and fake applications. However, Mezzour et al. do not examine malware types such as trojans, worms, and viruses. Finally, we find prior work that studies the correlation between users' demographics and attack encounters [22, 9, 24, 33, 38, 44], but does not statistically explain international differences.

In this paper, we examine international variation in the number of trojan, worm, and virus encounters and statistically test hypotheses about factors behind such variation. Such analysis allows policy actions aiming at reducing international malware encounters to rely on scientific empirical evidence instead of simply relying on expert opinions.

We extract the number of malware encounters in different countries from the Symantec Anti-Virus (AV) telemetry data. The AV telemetry data consist of threat reports collected from more than 10 million Symantec customer computers worldwide over the period November 2009 - September 2011. The AV telemetry data are accessible through the Symantec Worldwide Intelligence Network Environment (WINE) platform, which provides sampled, anonymized access to data collected from users that have opted in to report telemetry data from Symantec security products [16]. We use regression analysis to test for the relevance of various factors such as computing resources, web browsing behavior, 
cyber security expertise, computer piracy, and international relations.

We find that Sub-Saharan African countries are the most affected countries by trojans, worms, and viruses. Many Asian countries are also very affected by malware. The regression analysis reveals that widespread computer piracy especially when combined with poverty is the main reason behind high malware encounters in these countries. In poor countries where piracy is common, users obtain pirated software from P2P networks and merchants that sell pirated CDs publicly with near-impunity. Such pirated software is very likely to contain malware. In rich countries where piracy is common, piracy also takes the form of sharing legitimately purchased software copies among friends, family, and co-workers. Such form of piracy is less likely to propagate malware. Surprisingly, the regression analysis reveals that web browsing behavior, cyber security expertise, and international relations have no significant effect. Our study has important policy implications. Mainly, reducing malware encounters in the most affected regions requires addressing computer piracy. Other policy actions such as providing cyber security training or establishing cyber security institutions are unlikely to be effective if the piracy problem is not addressed.

The remainder of the paper is organized as follows. Section 2 discusses related work, Section 3 provides background, Section 4 presents the data, and Section 5 discusses threats to validity. Section 6 contains a descriptive analysis of the variation in the number of malware encounters across countries and Section 7 contains an explanatory analysis of that variation. Finally, Section 8 presents future work and Section 9 concludes.

\section{RELATED WORK}

The most closely related work is by Mezzour et al. [27] which tests hypotheses about factors behind international variation in the number of network-based attacks encountered and hosted. That work uses Symantec's Intrusion Prevention System (IPS) telemetry data which mainly include web attacks, fake applications (mainly fake anti-viruses), and exploits. Unfortunately, however, Mezzour et al. do not examine malware such as trojans, worms, and viruses. Other work that examines international variation in the number of attacks provides plausible explanations for this variation, but does not empirically test the validity of these explanations. For example, Caballero et al. [8] developed a malware measurement infrastructure and deployed it across 15 countries. The malware measurement infrastructure was used to interact with 4 Pay-Per-Install providers (one type of malware distribution services). Caballero et al. found that fake anti-viruses tend to target Western European and Northern American countries, and conjecture that this is because these countries have abundant monetary resources. Similarly, many security vendors e.g. Microsoft [30], McAfee [26], and Akamai [1] use their corporate data to create reports that depict international variation in the number of attacks. These reports, however, do not statistically test hypotheses about factors behind such international variation.

Moreover, several studies [22, 9, 24, 33, 38, 44] examine the relationship between users' demographics and/or behavior, and malware exposure. That type of user-level analysis is important, but does not provide insight into how various country-level technical, social, economic, and policy factors affect countries' exposure to malware. Understanding the effect of these country-level factors provides an opportunity for large scale reduction in the number of malware encounters. Some of these studies [22, 24] do not examine the effect of country-level characteristics because of the limited international span of their user base. For example, the study by Levesque et al. [22] uses data from 50 subjects in the Montreal area whose laptops were set up to gather data about malware infections and users' behavior. Maier et al. [24] use network traffic data of DSL users from an American university, an European urban area, and an Indian rural area. Covering 3 countries does not allow for the type of global analysis we perform in this paper. Other studies use social networking platforms [33] or Mechanical Turk [38] to recruit users, but do not report information about these users' countries. Finally, some studies $[9,44]$ have a wide international coverage, but have a different focus than empirically identifying factors behind international variation in the number of malware encounters. For example, Canali et al. [9] use Symantec data with wide international coverage to explore the possibility of predicting users' risk of encountering malicious web pages based on users' web browsing behavior. Yen et al. [44] use data about malware encounters in a large multi-national to predict the likelihood that hosts encounter malware based on the characteristics of these hosts' users.

\section{BACKGROUND}

In this section, we discuss factors that may impact the number of malware encounters in different countries. We choose such factors based on observations and hypotheses discussed in prior work.

Computing and monetary resources. The majority of attacks nowadays have a monetary goal. Therefore, we expect the availability of large computing and monetary resources to cause an increase in malware encounters. For example, Caballero et al. [8] notice that fake anti-viruses are most prevalent in Western Europe and North America, and hypothesize that this is due to the large resources in these countries. Moreover, the statistical analysis by Mezzour et al. [27] concludes that large resources increase encounters of web attacks, fake applications, and exploits.

Cyber security expertise. Prior work contains seemingly contradictory evidence about the effect of cyber security expertise. Onarlioglu et al. [33] perform a controlled experiment where experts and non-experts encounter the same attack scenarios. Onarlioglu et al. find that experts are more likely than non-experts to avoid sophisticated attack scenarios. On the other hand, Levesque et al. [22] and Yen et al. [44] find that experts encounter more malware than nonexperts when these experts and non-experts perform their daily activities on their own computers. The findings by Levesque et al. and Yen et al. are probably due to the fact that experts use their computers for longer periods of time and to perform more sophisticated tasks. 
Table 1: Example of a threat report

\begin{tabular}{ll}
\hline Field & Value \\
\hline Threat name & W32.Aimdes.A@mm \\
IP address & 259.23.78.45 \\
machine ID & 12:AC:54:BA:12:78 \\
\hline
\end{tabular}

Web browsing. When browsing the web, users may encounter a web attack that triggers a drive-by-download of malware [35]. Canali et al. [9] find that the number of web pages visited as well as the diversity of these pages (as measured by the number of top level domains visited) are correlated with the number of web attack encountered.

Computer piracy. Cyber criminals use pirated software to spread malware [21]. End-users obtain pirated software for the functionality, but often become infected as a result [21]. Therefore, we expect computer piracy to cause an increase in malware encounters.

International relations. Countries that have many international hostilities may be the target of sophisticated malware such as Flame and Stuxnet [32]. Similarly, countries with many military allies may be less likely to encounter malware since countries usually refrain from attacking their allies. Finally, countries that sign extradition treaties with more countries may encounter less malware because these countries may be able to prosecute cyber criminals from a wide range of countries.

\section{DATA}

\subsection{Malware Encounters}

In this section, we explain how we use the AV telemetry data [16] to compute the average number of trojans, worms, and viruses encountered by computer in each country. The AV telemetry data consist of threat reports from more than 10 million Symantec customer computers worldwide. The $\mathrm{AV}$ is an end-host system that detects malicious files on computers. Upon detecting a malicious file, the AV quarantines the file and sends a threat report to Symantec. Such threat report contains the threat name, and the reporting machine's IP address and unique identifier. A threat report example is given in Table 1 . We use the IP address to identify the computer's country [25]. IP geolocation is very accurate at the country level. We use the unique identifier to distinguish between victim computers.

We complement the AV telemetry data by a threat cata$\log [28]$ that contains structured descriptions of threats reported in the AV telemetry data. The threat catalog is extracted from Symantec's online threat descriptions [40]. An threat catalog entry contains the threat name, the threat family name, and the threat type. An example of such entry is given in Table 2. The threat name is the unique name that Symantec assigns to the threat, the threat family name is a generalization of the threat name, and the threat type can be trojan, worms, virus, or adware/spyware, etc. The main types in the AV telemetry data are trojans, worms, and viruses as depicted in Figure 1.
Table 2: Example of a threat catalog entry

\begin{tabular}{ll}
\hline Field & Value \\
\hline Threat name & W32.Aimdes.A@mm \\
Threat family name & Aimdes \\
Type & worm \\
\hline
\end{tabular}

Figure 1: Percentage of different threat types in the AV telemetry data

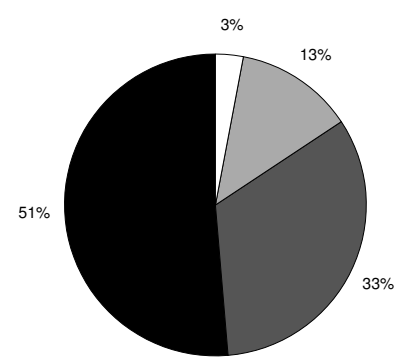

Trojans $\square$ Worms $\square$ Viruses $\square$ Other

The Symantec customer computers whose threat reports are in the data are randomly sampled among all Symantec customer computers worldwide using a sampling strategy that ensures that the WINE data are representative of all data collected by Symantec [34]. This work covers all 184 countries that have at least 30 Symantec customer computers included in the data. Countries excluded because they have less than 30 of these computers are North Korea, Nauru, Guinea-Bissau, Tuvalu, Eritrea, Cuba, and Kiribati. Figure Appendix.1 shows the number of Symantec customer computers included in the telemetry data for all 184 countries we cover in our analysis. As expected, developed countries have more Symantec customer computers than developing countries simply because developed countries have more computers in general.

We define the number of trojans encountered per computer, the number of worms encountered per computer, and the number of viruses encountered per computer in a country as the average number of unique threat families of type trojan, worm, and virus respectively that Symantec customer computers in that country encounter. Similarly, we define the number of all types encountered per computer in a country as the average number of unique threat families that Symantec customer computers in that country encounter.

We use the average number of malware encountered by Symantec customer computers in a country instead of the total number of malware encountered by these computers in order to allow for a meaningful comparison between countries with different numbers of Symantec customer computers. Moreover, we count the unique number of threat families instead of the number of threat reports because a computer may send multiple threat reports over time about the same infection.

We now consider a toy example in order to illustrate how we compute these measures. Assume that in a certain coun- 
try there are only two Symantec customer computers $A$ and B. $A$ sends 1 threat report about W32.Aimdes.A@amm (of threat family Aimdes and type worm) and 1 threat report about trojan.Tellafriend (of threat family Tellafriend and type trojan.), $B$ sends 1 threat report about trojan. Tellafriend and 5 threat reports about threat Ada (of threat family Ada and type virus.) The number of trojans encountered per computer in the country is 1 , the number of worms encountered per computer is $1 / 2$, the number of viruses encountered per computer is $1 / 2$, and the number of all types encountered per computer is 2 .

\subsection{Explanatory Variables}

In this section, we explain how we measure explanatory factors discussed in Section 3.

Computing and monetary resources. In order to estimate the strength of computing resources in a country, we use the ICT development index and the Internet bandwidth measure from the International Telecommunication Union branch of the United Nation [20]. We estimate the richness of people in a country using the GDP per capita $[41,12]$.

Cyber security expertise. Measuring the average security expertise of a country's users is difficult. As a proxy, we use the strength of cyber security research and the existence of cyber security institutions such as CERTs in the country.

We estimate the strength of cyber security research in a country by counting the number of cyber security research papers that the country wrote during the period 2002-2011. We take into account research papers published over 10 years because expertise gained in research takes time to transfer to the general public. We collect from SCOPUS [37] all 28,400 research papers that contain "security" in their title or abstract and that belong to the computer science or engineering areas. We consider that a country wrote a paper if at least one of the authors has an affiliation in that country.

We obtain the list of countries that have cyber security institutions by combining lists from multiple sources $[23,13$, 19]. We construct a binary variable that captures whether a country has such institutions.

Web browsing. We use the average number of unique web pages visited (hits) and the average number of top level domains visited (tops) in a country from Canali et al. [9]. Canali et al. compute these estimates based on data collected from a subset of Symantec customers that agree to share their web browsing histories with Symantec.

Computer piracy. We use the piracy rate from the Business Software Alliance (BSA) [7]. The piracy rate in a country is the ratio of the number of unlicensed software units installed to the total number of software units installed. The BSA collects such data by surveying users in different countries about their practices.
International relations. Based on the list of military and non-military hostilities [11, 15] during the period 1992-2010, we construct a binary country-by-country hostility network $H=\left[h_{i j}\right]$ where $h_{i j}=1$ indicates the existence of a hostility between $i$ and $j$, and $h_{i j}=0$ indicates otherwise. We then compute hostility betweenness as countries' betweenness centrality in that network. Betweenness centrality [17] is a standard measure of the importance of a node in a network. More specifically, betweenness centrality measures the extent to which a node falls in shortest paths among other nodes.

We proceed similarly for international alliances and extraditions. We construct a binary alliance network and a binary extradition network based on the list of international military alliances [14] and the list of international extradition treaties [43] respectively. We then compute alliance betweenness and extradition betweenness as countries' betweenness centrality in the alliance network and extradition network respectively.

\section{THREATS TO VALIDITY}

The limitations of this paper mainly stem from limitations of the data we use. First, Symantec AV data inform about malware encounters of Symantec AV home users. Malware encounters of home users of other paid-for AV products have no reasons to be different. However, malware encounters of corporate users, users that use no AV protection or users that use free AV products are probably different. Unfortunately, we are unable to correct for this bias because we are unaware of any study that compares malware encounters of different types of users at a global scale. Moreover, the data cover the time period 2009-2011. The malware landscape may have changed since then. For example, as the IT infrastructure in African countries have improved over time, these countries may be encountering even more malware as cyber-criminals may become even more interested in attacking these countries. Furthermore, the Symantec AV telemetry data only cover malware detected by Symantec AV. Sophisticated malware such as targeted attacks and zero-day attacks are missing in the data. It is worth noting, however, that targeted attacks and zero-day attacks are a small minority compared with other attacks [5, 42].

It is also worth mentioning that the Symantec labeling of attacks that we use in this paper is not perfect. Inconsistencies among AV vendors in labeling malware have been reported $[3,10,31]$. However, we believe that the lack of a unified malware labeling taxonomy is the main reason behind such inconsistencies. The fact that different AV vendors label malware differently does not necessarily indicate that the labeling from any AV vendor is wrong. Many research papers $[4,18,36]$ use AV labels as a ground-truth when evaluating the accuracy of new approaches.

Finally, we rely on IP geolocation to identify countries where computers are. Threats to validity that usually come with using IP geolocation are IP spoofing and the use of anonymization software. We do not believe that IP spoofing is an issue for our data given Symantec customer computers have no reason to spoof their address when sending threat reports to Symantec. Similarly, the use of anonymization software such as Tor is unlikely to cause a significant bias because only a 
small minority of the general population uses such software. Pirated software users also typically do not use anonymization software. In places such as Sub-Saharan Africa, pirated software is sold publicly in the streets and there is no penalty for using such software that would cause users to hide their identity using anonymization software [6].

\section{DESCRIPTIVE ANALYSIS}

Table 3 contains the list of countries that rank highest on the number of malware encounters. We see that these countries are primarily poor countries in Sub-Saharan Africa. This is surprising given that Caballero et al. [8] and Mezzour et al. [27] find that fake applications and web attacks are most prevalent in Western Europe and North America. This finding probably indicates that factors that drive encounters of trojans, worms, and viruses are very different from factors that drive encounters of web attacks and fake applications.

Figure 2 presents a map visualization of the number of malware encounters in all countries. We see that Sub-Saharan Africa encounters the largest quantities of trojans, followed by Asia and North Africa. Other regions encounter relatively small quantities of trojans. Moreover, we see that Sub-Saharan Africa is also the most affected region by worms and viruses, followed by South-East Asia and South Asia. Other regions encounter relatively small quantities of worms and viruses. Finally, when taking into consideration all malware types, we see a pattern that is similar to the pattern of trojans. This is due to the fact that trojans constitute the majority of malware types in the data as depicted in Figure 1.

\section{EXPLANATORY ANALYSIS}

The goal of this section is to identify factors that explain international variation in malware encounters. In order to identify these factors, we use regression analysis which aims at establishing causation, and not just correlation. Table 4 contains summary statistics of the variables we use in this section. Each variable is of length 184, the number of all countries in the world that have at least 30 Symantec customer computers whose threat reports are included in the AV telemetry data as we explain in Section 4.1. Trojans encountered per computer, worms encountered per computer, viruses encountered per computer, and all types encountered per computer are the dependent variables we will use in the regression analysis. These variables represent the average number of trojans, worms, viruses, and all malware types respectively encountered by a single Symantec customer computer ${ }^{1}$ as explained in Section 4.1. Other variables are the explanatory variables we intend to use in the regression analysis and that were explained in Section 4.2.

Table 5 represents the correlation table between the explanatory variables. Each element in the table represents the cor-

\footnotetext{
${ }^{1}$ The careful reader will notice that all types encountered per computer is not the sum of trojans, worms, viruses and other types encountered per computer. This is because these measures are scaled by the number of Symantec customer computers in each country. If we were to consider the unscaled measures, we would find that all types encountered by all computers in the world is the sum of trojans, worms, viruses, and other types encountered by all computers in the world.
}

relation between two explanatory variables. For example, the correlation between ICT and bandwidth is 0.60 . In the correlation table, we only report values below the diagonal because correlation is symmetric. For example, the correlation between GDP PC and ICT is the same as the correlation between ICT and GDP PC. From Table 5, we see that GDP $\mathrm{PC}$ is highly correlated with ICT, that is countries with high income per individual tend to have strong computing infrastructure. Because of this very high correlation value (0.92), we only use one of these variables in the regression analysis. From the table, we also see that computer piracy is more prevalent in countries with low income. This reflects the fact that low-income individuals are unable to afford to legitimate software and music, and resort to pirated products instead.

Table 6 presents the results of the regression analysis. From the table, we see that the main factor that causes high malware encounters is high piracy rates especially when these high piracy rates are combined with low computing and monetary resources. In poor countries where piracy is common, people obtain pirated software and music through $\mathrm{P} 2 \mathrm{P}$ networks and merchants that sell these products publicly in the streets with near-impunity [6]. These merchants obtain pirated products and license keys from dubious Internet websites. As a result, pirated software in these poor countries is very likely to contain malware. On the other hand, in relatively rich countries where piracy is common such as Singapore, piracy also takes the form of sharing legitimately purchased products among friends, family, and co-workers because the collectivism culture encourages sharing and helping others [39, 2]. This form of piracy is less likely to spread malware.

From Table 6, we also see that web browsing behavior has surprisingly no significant effect on international variation in the number of malware encounters. Web browsing has no significant impact because, as explained in the previous paragraph, computers encounter malware primarily through pirated computer products, and not through web attacks and drive-by-downloads. Cyber security expertise, too, has surprisingly no significant effect. Many people are aware that pirated products are less safe [7], but prefer such pirated products because of economic, social, and legal reasons [7]. Finally, it is surprising that international relations have no significant effect. Despite the wide media coverage of state-sponsored malware, the volume of such malware is very small compared with criminally motivated malware [42]. Moreover, state-sponsored malware tends to be very sophisticated and is often undetected by anti-viruses.

\section{FUTURE WORK}

As future work, it would be interesting to examine measures beyond the average number of malware encounters per computer. For example, it would be interesting to examine the median and the ratio of Symantec customer computers that encounter malware out of all Symantec customer computers in a country. It would also be interesting to use a stepwise approach such as the Akaike Information Criterion (AIC) and Bayesian Information Criteria (BIC) approach in the regression analysis.

Another interesting future work direction is to examine the 
Table 3: Top 10 countries on the number of malware encounters per computer

\begin{tabular}{ll|ll|ll|ll}
\hline Country & \# trojans & Country & \# worms & Country & \# viruses & Country & \# all types \\
\hline Burundi & 1.56 & Solomon Islds & 8.76 & Chad & 1.99 & Solomon Islds & 17.51 \\
Solomon Islds & 1.43 & Bhutan & 5.94 & Somalia & 1.78 & Swaziland & 13.14 \\
Bhutan & 1.24 & Cent. Afr. R. & 5.55 & Burundi & 1.64 & Bhutan & 13.09 \\
Ethiopia & 1.20 & Swaziland & 5.38 & Cent. Afr. R. & 1.58 & Chad & 11.78 \\
Cambodia & 1.19 & Chad & 5.36 & Swaziland & 1.57 & Cent. Afr. Rep. & 11.68 \\
Yemen & 1.19 & Samoa & 5.05 & Solomon Islds & 1.36 & Burundi & 11.10 \\
Cent. Afr. R. & 1.18 & Somalia & 4.68 & Gambia & 1.29 & Somalia & 10.84 \\
Swaziland & 1.18 & Burundi & 4.46 & Togo & 1.28 & Samoa \\
Chad & 1.17 & Sierra Leone & 4.05 & Cameroon & 1.23 & Sierra Leone & 9.88 \\
Syria & 1.13 & Lesotho & 3.77 & Sierra Leone & 1.23 & Togo & 9.08 \\
\hline
\end{tabular}

Table 4: Summary statistics of variables used in the explanatory analysis

\begin{tabular}{lccccc}
\hline \hline Statistic & $\mathrm{N}$ & Mean & St. Dev. & Min & Max \\
\hline Trojans encountered per computer & 184 & 0.55 & 0.25 & 0.22 & 1.56 \\
Worms encountered per computer & 184 & 1.42 & 1.34 & 0.045 & 8.76 \\
Viruses encountered per computer & 184 & 0.34 & 0.39 & 0.031 & 1.99 \\
All types encountered per computer & 184 & 4.06 & 2.64 & 0.94 & 17.51 \\
& & & & \\
Bandwidth & 184 & 30.95 & 57.66 & 0.10 & 547.10 \\
ICT & 184 & 3.82 & 2.05 & 0.85 & 8.45 \\
GDP per capita (log) & 184 & 8.49 & 1.53 & 5.29 & 11.55 \\
Web hits & 184 & $1,020.52$ & 586.86 & 105 & 5,363 \\
Web tops & 184 & 12.89 & 4.11 & 1.00 & 32.33 \\
Piracy & 184 & 0.65 & 0.21 & 0.20 & 0.93 \\
Security research & 184 & 175.50 & 830.41 & 0 & 7,911 \\
Security institutions & 184 & 0.36 & 0.48 & 0 & 1 \\
Alliance betweenness & 184 & 0.001 & 0.007 & 0 & 0.053 \\
Hostility betweenness & 184 & 0.0004 & 0.002 & 0 & 0.017 \\
Extradition betweenness & 184 & 0.004 & 0.036 & 0 & 0.483 \\
\hline \hline
\end{tabular}

Table 5: Correlation table between explanatory variables

\begin{tabular}{|c|c|c|c|c|c|c|c|c|c|c|}
\hline & Bandwidth & ICT & GDP PC & web hits & web tops & piracy & research & institutions & alliance & hostility \\
\hline $\mathrm{ICT}$ & $0.60^{* * *}$ & & & & & & & & & \\
\hline GDP PC & $0.52^{* * *}$ & $0.92 * * *$ & & & & & & & & \\
\hline web hits & 0.13 & $0.25^{* * *}$ & $0.24^{* *}$ & & & & & & & \\
\hline web tops & -0.04 & 0.02 & 0.00 & $0.65^{* * *}$ & & & & & & \\
\hline piracy & $-0.54 * * *$ & $-0.85 * * *$ & $-0.85 * * *$ & $-0.23^{* *}$ & 0.04 & & & & & \\
\hline research & 0.09 & $0.23^{* *}$ & $0.20^{* *}$ & 0.10 & -0.10 & $-0.24 * *$ & & & & \\
\hline institutions & $0.34 * * *$ & $0.56^{* * *}$ & $0.47^{* * *}$ & 0.14 & -0.03 & $-0.49 * * *$ & $0.27 * * *$ & & & \\
\hline alliance & 0.10 & $0.21^{* *}$ & $0.18^{*}$ & 0.06 & -0.06 & $-0.21 * *$ & $0.46 * * *$ & $0.20 * *$ & & \\
\hline hostility & 0.03 & 0.02 & 0.00 & 0.00 & -0.01 & -0.03 & $0.38 * * *$ & 0.07 & $0.30 * * *$ & \\
\hline extradition & 0.03 & 0.13 & 0.12 & 0.06 & -0.05 & $-0.19^{*}$ & $0.70^{* * *}$ & 0.12 & $0.58^{* * *}$ & $0.55^{* * *}$ \\
\hline
\end{tabular}




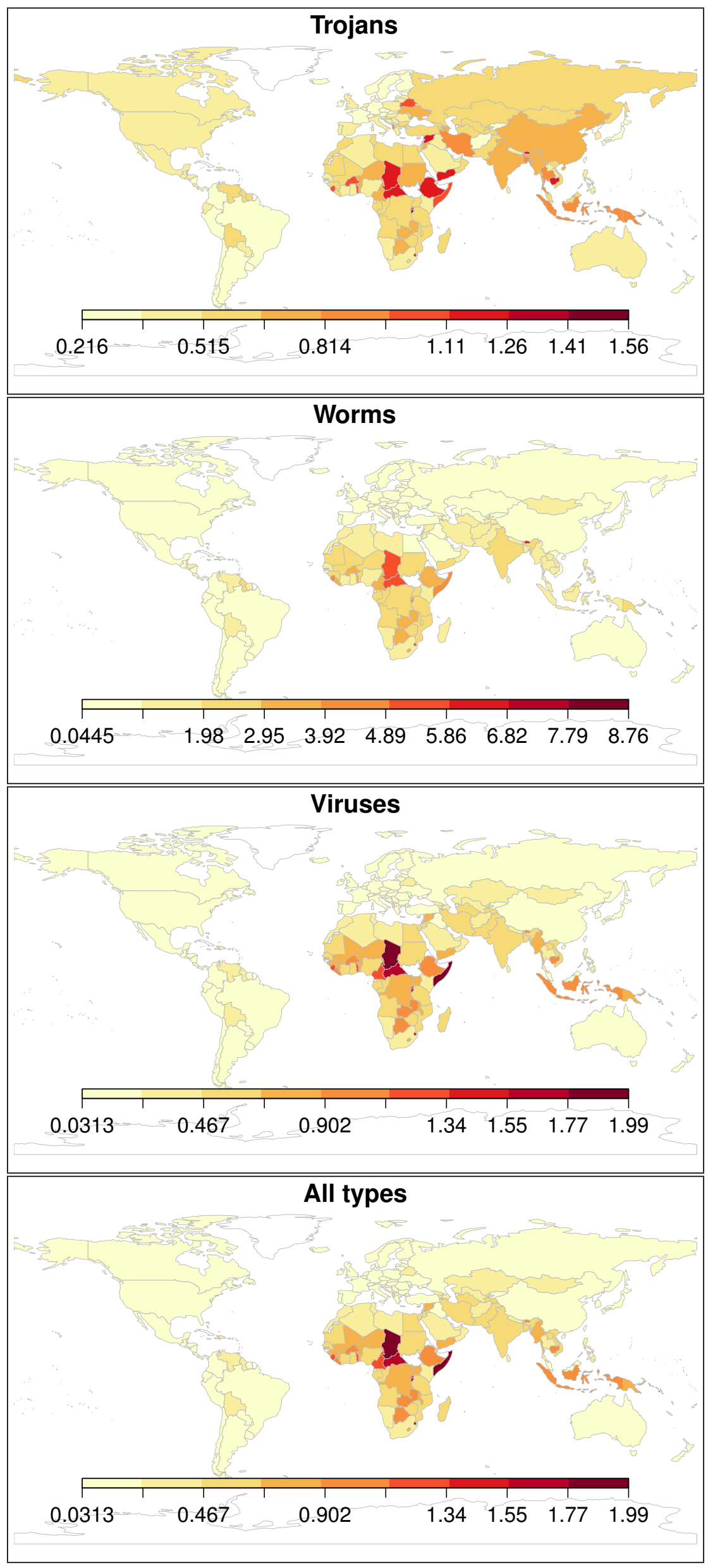

Figure 2: Map visualization of the number of threats encountered per computer 
Table 6: Regression Analysis of the number of malware encounters in different countries. Regression coefficients are standardized.

\begin{tabular}{|c|c|c|c|c|}
\hline & Trojans & Worms & Viruses & All types \\
\hline \multicolumn{5}{|c|}{ Computing and monetary resources } \\
\hline Bandwidth & $\begin{array}{l}-0.010 \\
(0.081)\end{array}$ & $\begin{array}{l}0.031 \\
(0.069)\end{array}$ & $\begin{array}{l}0.013 \\
(0.060)\end{array}$ & $\begin{array}{l}0.022 \\
(0.070)\end{array}$ \\
\hline $\mathrm{ICT}$ & $\begin{array}{l}-0.068 \\
(0.285)\end{array}$ & $\begin{array}{l}-0.387 \\
(0.242)\end{array}$ & $\begin{array}{l}0.302 \\
(0.211)\end{array}$ & $\begin{array}{l}-0.313 \\
(0.247)\end{array}$ \\
\hline \multicolumn{5}{|l|}{ Web browsing } \\
\hline Web hits & $\begin{array}{l}0.026 \\
(0.087)\end{array}$ & $\begin{array}{l}0.036 \\
(0.074)\end{array}$ & $\begin{array}{l}0.021 \\
(0.064)\end{array}$ & $\begin{array}{l}0.032 \\
(0.075)\end{array}$ \\
\hline Web tops & $\begin{array}{l}0.090 \\
(0.085)\end{array}$ & $\begin{array}{l}0.018 \\
(0.072)\end{array}$ & $\begin{array}{l}0.024 \\
(0.063)\end{array}$ & $\begin{array}{l}0.047 \\
(0.074)\end{array}$ \\
\hline \multicolumn{5}{|l|}{ Piracy } \\
\hline Piracy & $\begin{array}{l}0.493^{* *} \\
(0.222)\end{array}$ & $\begin{array}{l}0.149 \\
(0.189)\end{array}$ & $\begin{array}{l}0.813^{* * *} \\
(0.164)\end{array}$ & $\begin{array}{l}0.282 \\
(0.193)\end{array}$ \\
\hline Piracy x ICT & $\begin{array}{c}-0.244^{*} \\
(0.145)\end{array}$ & $\begin{array}{l}-0.299^{* *} \\
(0.123)\end{array}$ & $\begin{array}{l}-0.684^{* * *} \\
(0.107)\end{array}$ & $\begin{array}{l}-0.285^{* *} \\
(0.125)\end{array}$ \\
\hline \multicolumn{5}{|c|}{ Security research and institutions } \\
\hline Security research & $\begin{array}{l}0.082 \\
(0.090)\end{array}$ & $\begin{array}{l}-0.059 \\
(0.077)\end{array}$ & $\begin{array}{l}-0.085 \\
(0.067)\end{array}$ & $\begin{array}{l}-0.043 \\
(0.078)\end{array}$ \\
\hline Security institutions & $\begin{array}{l}0.079 \\
(0.076)\end{array}$ & $\begin{array}{l}-0.070 \\
(0.065)\end{array}$ & $\begin{array}{l}0.056 \\
(0.056)\end{array}$ & $\begin{array}{l}-0.010 \\
(0.066)\end{array}$ \\
\hline \multicolumn{5}{|c|}{ International relations } \\
\hline Alliance betweenness & $\begin{array}{l}0.016 \\
(0.077)\end{array}$ & $\begin{array}{l}0.011 \\
(0.065)\end{array}$ & $\begin{array}{l}0.009 \\
(0.057)\end{array}$ & $\begin{array}{l}0.011 \\
(0.066)\end{array}$ \\
\hline Hostility betweenness & $\begin{array}{l}0.023 \\
(0.074)\end{array}$ & $\begin{array}{l}0.010 \\
(0.063)\end{array}$ & $\begin{array}{l}0.054 \\
(0.055)\end{array}$ & $\begin{array}{l}0.016 \\
(0.064)\end{array}$ \\
\hline Extradition betweenness & $\begin{array}{l}-0.023 \\
(0.104)\end{array}$ & $\begin{array}{l}0.013 \\
(0.088)\end{array}$ & $\begin{array}{l}0.015 \\
(0.077)\end{array}$ & $\begin{array}{l}0.014 \\
(0.090)\end{array}$ \\
\hline Observations & 184 & 184 & 184 & 184 \\
\hline $\mathrm{R}^{2}$ & 0.352 & 0.534 & 0.647 & 0.514 \\
\hline
\end{tabular}

Standard errors in parentheses

${ }^{*} \mathrm{p}<0.1 ;{ }^{* *} \mathrm{p}<0.05 ;{ }^{* * *} \mathrm{p}<0.01$ 
relationship between encounters of network based attacks [27] and malware encounters. Similarly, it would be interesting to use data from other AV vendors. Finally, case studies about piracy and malware encounters in Sub-Saharan Africa would shed greater light into problematic practices in these countries and appropriate policy actions to address these practices.

\section{CONCLUSION AND DISCUSSION}

In this paper, we empirically test hypotheses about factors behind international variation in the number of malware encounters. Such hypothesis testing provides an empirical scientific basis to our understanding of global malware encounters and to policy actions to reduce such encounters. To the best of our knowledge, this is the first research paper that statistically tests hypotheses about reasons behind international differences in the number of trojans, worms, and viruses encountered.

We extract the number of malware encounters in each country from the Symantec AV telemetry data. That data consist of threat reports from more than 10 million Symantec customer computers worldwide. We use regression analysis to test for the relevance of computing and monetary resources, web browsing behavior, computer piracy, security expertise, and international relations.

We find that trojans, worms, and viruses are most prevalent in Sub-Saharan Africa. The regression analysis reveals that this is mainly due to widespread computer piracy combined with poverty in this region. In poor countries where piracy is widespread, users obtain pirated computers products from $\mathrm{P} 2 \mathrm{P}$ networks and merchants that sell pirated CDs on the streets with near impunity. These merchants obtain these pirated products from dubious parts of the Internet. On the other hand, in rich countries where piracy is widespread, it is more common for a person to buy legitimate software or music and share it with friends, family, and coworkers.

The regression analysis reveals many surprising findings. For example, given that malware have primarily a monetary goal, we hypothesized that cyber criminals would target rich countries because the monetary benefit of compromising rich people's computers is higher. Our hypothesis is consistent with what Caballero et al. [8] and Mezzour et al. [27] observed about fake anti-viruses and other types of network based attacks. In this paper, we found that, surprisingly, poor countries in Sub-Saharan Africa are most exposed to malware. One possible explanation is that cyber criminals target computers in Sub-Saharan Africa because of the low cost of attacking computers in this region. Another possible explanation is that cyber-criminals do not target this region on purpose. It is just that one malware distribution method, mainly computer piracy, happens to be particularly popular in Sub-Saharan Africa.

Our work has many policy implications. Reducing malware encounters in Sub-Saharan Africa requires reducing computer piracy in this region. Other policy actions such as providing cyber security training are unlikely to be effective. Software and music industries are the entities that have the highest incentive to combat computer piracy in order to increase revenues. Governments in this region are aware that legitimate software is more stable and safer than pirated software. However, these governments are reluctant to fight computer piracy despite lobbying by the software industry because such fight would prevent their populations from keeping up with international knowledge and technology. For example, the GDP per capita is 700 USD in Central African Republic [12], while Mircosoft Windows costs 119 USD [29]. It is unreasonable to believe that poor people would pay more than $15 \%$ of their yearly income in order to acquire a legitimate copy of Microsoft Windows. We join Gobal [39] in suggesting that the software and music industries should adjust their international prices to countries' income level. This could be a win-win solution because the software industry would be able to collect some revenue instead of losing almost all potential revenue to piracy and these countries' populations can have access to safe and robust computer products.

Meanwhile, given that malware can be "contagious", it is important to reduce the risk that malware from these countries spreads elsewhere. First, global organizations with offices in the most at-risk countries should have very strong, enforced, and enforceable policies for monitoring for, and correcting for malware. Second, those traveling to the most at-risk countries should be particularly wary of linking their own machines to machines in these countries and transferring material from the machines in these countries without having sufficient malware detectors and barriers in place. Third, soft-power solutions that raise awareness about the issue and provide anti-viruses to these countries could reduce the overall global risk.

\section{ACKNOWLEDGMENTS}

We would like to thank Matthew Elder, Tudor Dumitras and William Frankenstein for useful feedback and discussions. Tudor also provided critical support prior to and during our visit to Symantec Research Labs to access the AV telemetry data. Finally, we would like to thank Symantec for granting us access to the AV telemetry data. Data about malware encounters are accessible from Symantec using the reference WINE 2012 004. Explanatory variables are available from the authors upon request.

This work is supported in part by the Defense Threat Reduction Agency (DTRA) under grant HDTRA11010102, and the Army Research Office (ARO) under grants

ARO W911NF1310154 and ARO W911NF0910273, and the center for Computational Analysis of Social and Organizational Systems (CASOS). The views and conclusions contained in this document are those of the authors and should not be interpreted as representing the official policies, either expressed or implied, of DTRA, ARO or the U.S. government.

\section{REFERENCES}

[1] Akamai. Akamai's state of the internet report, Q1 2014.

[2] K. Bagchi, P. Kirs, and R. Cerveny. Global software piracy: can economic factors alone explain the trend? Communications of the ACM, 49(6):70-76, June 2006.

[3] M. Bailey, J. Oberheide, J. Anderen, Z. M. Mao, F. Jahanian, and J. Nezario. Automated classification and analysis of internet malware. In International 
Symposium on Research in Attacks, Instrusions and Defenses (RAID), September 2007.

[4] U. Bayer, P. M. Comparetti, C. Hlauschek, C. Kruegel, and E. Kirda. Scalable, behavior-based malware clustering. In Network and Distributed System Security Symposium (NDSS), San Diego, CA, February 2009.

[5] L. Bilge and T. Dumitraş. Before we knew it. An empirical study of zero-day attacks in the real world. In Computer and Communication Security Conference (CCS), Raleigh, NC, October 2012.

[6] BIZTECH AFRICA. Nigeria at the mercy of software pirates. http://www.biztechafrica.com/article/ nigeria-mercy-software-pirates/3871/\#. VLrP9nv0_Sg, 2012.

[7] Business Software Alliance. 2010 piracy study. Technical report, May 2011.

[8] J. Caballero, C. Grier, C. Kreibich, and V. Paxson. Measuring pay-per-install: The commoditization of malware distribution. In The $20^{\text {th }}$ USENIX Security Symposium, San Francisco, CA, August 2011.

[9] D. Canali, L. Bilge, and D. Balzarotti. On the effectiveness of risk prediction based on users browsing behavior. In ACM symposium on Information, computer and communications security (ASIA CCS), pages 171-182. ACM Press, 2014.

[10] J. Canto, M. Dacier, E. Kirda, and C. Leita. Large scale malware collection: Lessons learned. In IEEE SRDS Workshop on Sharing Field Data and Experiment Measurements on Resilience of Distributed Computed Systems, October 2008.

[11] Center for International Development and Conflict Management. International crisis behavior project. http://www. cidcm.umd.edu/icb/. Last accessed: December 2011.

[12] Central Intelligence Agency. The World Factbook. https://www.cia.gov/library/publications/ the-world-factbook/. Last accessed: January 2015.

[13] CERT. National computer security incident response teams. http:

//www.cert.org/csirts/national/contact.html, 2014. Last accessed: January 2014.

[14] Correlates of War Project. Alliances v3.03. http: //www . correlatesof war . org/. Last accessed: December 2011.

[15] Department of Peace and Conflict Research. Uppsala University. Ucdp dyadic dataset. http://www.pcr.uu. se/research/ucdp/datasets/ucdp_dyadic_dataset/. Last accessed: December 2011.

[16] T. Dumitras and D. Shou. Toward a standard benchmark for computer security research. The worldwide intelligence network environment (WINE). In Workshop on Building Analysis Datasets and Gathering Experience Returns for Security (BADGERS), Salzburg, Austria, April 2011.

[17] L. C. Freeman. A Set of Measures of Centrality Based on Betweenness. Sociometry, 40(1):35, Mar. 1977.

[18] X. Hu, T. Chiueh, and K. G. Shin. Large-scale malware indexing using function-call graphs. In Computer and Communication Security Conference (CCS), Chicago, IL, November 2009.

[19] International Cyber Center. George Mason University.
Certicc home.

http://internationalcybercenter .org/certicc, 2014. Last accessed: January 2014.

[20] International Telecommunication Union. Measuring the information society. http://www.itu.int/en/ ITU-D/Statistics/Documents/publications/ mis2012/MIS2012_without_Annex_4.pdf, 2012.

[21] M. Kammerstetter, C. Platzer, and G. Wondracek. Vanity, cracks and malware: insights into the anti-copy protection ecosystem. In Computer and Communication Security Conference (CCS), 2012.

[22] F. Lalonde Levesque, J. Nsiempba, J. M. Fernandez, S. Chiasson, and A. Somayaji. A clinical study of risk factors related to malware infections. In $A C M$ SIGSAC conference on Computer and communications security (CCS), pages 97-108, Berlin, Germany, November 2013. ACM Press.

[23] J. A. Lewis and K. Timlin. Cybersecurity and cyberwarfare. Preliminary assessment of national doctrine and organization. Technical report, Center for Strategic and International Studies, 2011.

[24] G. Maier, A. Feldmann, V. Paxson, R. Sommer, and M. Vallentin. An assessment of overt malicious activity manifest in residential networks. In Detection of Intrusions and Malware, and Vulnerability Assessment, volume 6739, pages 144-163. Springer Berlin Heidelberg, Berlin, Heidelberg, 2011.

[25] Maxmind. Geolite free downloadable databases. Geolite country.

http://dev.maxmind.com/geoip/legacy/geolite/, November 2012.

[26] McAfree. Mcafee labs threats report. http://www.mcafee.com/us/resources/reports/ rp-quarterly-threat-q1-2014.pdf, June 2014.

[27] G. Mezzour, L. R. Carley, and K. M. Carley. Global mapping of cyber attacks. Technical Report CMU-ISR-14-111, Carnegie Mellon University, School of Computer Science, Institute for Software Research, 2014.

[28] G. Mezzour, L. R. Carley, and K. M. Carley. Longitudinal analysis of a large corpus of cyber threat descriptions. Journal of Computer Virology and Hacking Techniques, June 2014.

[29] Microsoft. Windows 8.1. http://www.microsoftstore.com/store/msusa/en_ US/pdp/Windows-8.1/productID . 288401200, 2015. http://www.microsoftstore.com/store/msusa/en_ US/pdp/Windows-8.1/productID . 288401200.

[30] Micrsoft. Micrsoft security intelligence report. Worldwide threat assessment, July-December 2013.

[31] D. Moore, V. Paxson, S. Savage, C. Shannon, S. Staniford, and N. Weaver. Inside the slammer worm. IEEE Security and Privacy, 4(1):33-39, July 2003.

[32] New York Times. Obama calls for new law to bolster cybersecurity. http://www.nytimes.com/2015/01/14/us/ obama-to-announce-new-cyberattack-protections. html?_r=0, 2015.

[33] K. Onarlioglu, Y. O. Yilmaz, E. Kirda, and D. Balzarotti. Insights into user behavior in dealing with internet attacks. In Network and Distributed 
System Security Symposium (NDSS), San Diego, CA, Feb. 2012.

[34] E. E. Papalexakis, T. Dumitras, D. H. P. Chau, B. A. Prakash, and C. Faloutsos. Spatio-temporal mining of software adoption \&amp; penetration. In IEEE/ACM International Conference on Advances in Social Networks Analysis and Mining, pages 878-885. ACM Press, 2013.

[35] N. Provos, P. Mavrommatis, M. A. Rajab, and F. Monrose. All your iframes point to us. In 17 th Usenix Security Symposium, San Jose, CA, July 2008.

[36] K. Rieck, T. Holz, C. Willems, P. Düssel, and P. Laskov. Learning and classification of malware behavior. In Conference on Detection of Intrusions and Malware and Vulnerability (DIMVA), pages 108-125, Paris, France, July 2008.

[37] SCOPUS. www. scopus. com. Last accessed: October 2012.

[38] S. Sheng, M. Holbrook, P. Kumaraguru, L. F. Cranor, and J. Downs. Who falls for phish?: a demographic analysis of phishing susceptibility and effectiveness of interventions. In SIGCHI Conference on Human Factors in Computing Systems (CHI), page 373, Atlanta, GA, April 2010. ACM Press.

[39] S. K. Shin, R. D. Gopal, G. L. Sanders, and A. B. Whinston. Global software piracy revisited. Communications of the ACM, 47(1):103-107, Jan. 2004.

[40] Symantec threat explorer. http://www.symantec.com/ security_response/landing/azlisting.jsp. Last accessed: October 2012.

[41] The World Bank. World development indicators (wdi) 2012. http://data.worldbank.org/data-catalog/ world-development-indicators/wdi-2012, April 2012.

[42] O. Thonnard, L. Bilge, G. O'Gorman, S. Kiernan, and M. Lee. Industrial espionage and targeted attacks: Understanding the characteristics of an escalating threat. In International Symposium on Research in Attacks, Instrusions and Defenses (RAID), September 2012.

[43] United Nations Crime and Justice Information Network. Bilateral agreements on extradition, judicial/legal assistance, control of narcotic drugs, and prisoner transfer by country. http:

//www.uncjin.org/Laws/extradit/extindx.htm.

[44] T.-F. Yen, V. Heorhiadi, A. Oprea, M. K. Reiter, and A. Juels. An epidemiological study of malware encounters in a large enterprise. In $A C M S I G S A C$ conference on Computer and communications security (CCS), pages 1117-1130. ACM Press, 2014.

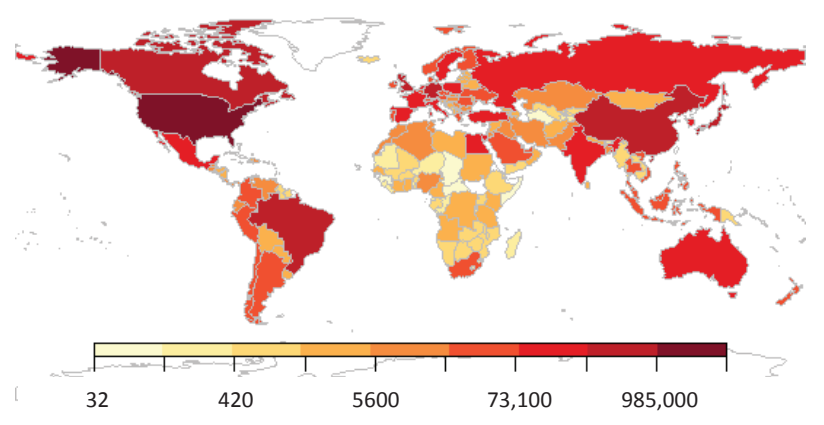

Figure Appendix.1: Number of Symantec customer computers included in the telemtry data for all countries included in the analysis

\section{APPENDIX}

[Number of Symantec customer computers] 